Case report

\title{
Metastatic pancreatic adenocarcinoma treated with liposomal irinotecan in combination with 5-fluorouracil and leucovorin as a II line chemotherapy
}

Leszek Kraj, Maciej Gryziak, Ewa Żurawińska-Grzelka, Krzysztof Woźniak

Clinic of Oncology, The Medical University of Warsaw

Correspondence: Leszek Kraj

Clinic of Oncology, The Medical University of Warsaw 02-097 Warszawa, Banacha 1A e-mail: leszek.kraj@wum.edu.pl

Received: 18.02.2021 Accepted: 27.06.2021

DOI: 10.24292/01.OR.122270621 Copyright $\odot$ Medical Education. All rights reserved.

\section{ABSTRACT}

The paper presents a case of a 54-year-old man with pancreatic tumor and intraperitoneal dissemination. The patient received treatment with gemcitabine in combination with nab-paclitaxel. After 18 months, the disease progressed, therefore the line of treatment was applied in the form of liposomal irinotecan with 5-FU/LV. This therapy provided progression-free survival for 7 months. The obtained results are better than the median progression-free survival obtained in the studies. This case demonstrates that liposomal irinotecan in the treatment of stage IV disease in patients progressing after gemcitabine opens up new treatment options.

Key words: II line chemotherapy, liposomal irinotecan, leucovorin, peripheral polyneuropathy, overall survival, pancreatic cancer 


\section{INTRODUCTION}

Despite the significant progress in the systemic treatment of many cancers, the therapy of patients with diagnosed pancreatic adenocarcinoma is still a huge challenge for modern oncology. 5-year survival rates for this patient population do not exceed $10 \%$ [1]. Pancreatic cancer is the fifth leading cause of death among all cancers. However, due to the increasing incidence, the lack of effective methods of prevention and limited therapeutic possibilities, it is estimated that by 2030 it may become even the second cause of death from malignant neoplasms in Europe [2] Therefore, there is an urgent need to search for and introduce new, more effective therapeutic methods.

Radical surgery followed by adjuvant chemotherapy is the only form of therapy that gives a chance for complete recovery. However, at the time of diagnosis of pancreatic cancer, only $10-15 \%$ of patients are eligible for radical therapy. In the remaining patients, it is impossible due to the local advancement (approx. 25\% of patients) or distant metastases (approx. 55\% of patients at diagnosis) [3].

Systemic chemotherapy is the primary treatment method in patients diagnosed with metastatic adenocarcinoma of the pancreas. Until recently, the median overall survival (OS) in this group of patients did not exceed 6 months, and the standard treatment was gemcitabine monotherapy [4]. Two additional treatment options have emerged in the last decade. In 2011, data from the phase III study on the efficacy of FOLFIRINOX were published. Its use was associated with an increase in median OS to 11.1 months compared with 6.8 months for gemcitabine monotherapy. There were also significant differences in progression free survival (PFS) and objective response rate (ORR), 6.4 months vs 3.3 months (median PFS) and 32\% vs 9\%, respectively (ORR) [5]. On the other hand, MPACT, a phase III randomized trial, verified the efficacy of nab-paclitaxel (paclitaxel with albumin) added to gemcitabine also compared to gemcitabine monotherapy. This study also reported significant increases in median OS (8.5 months vs 6.7 months), PFS (5.5 months vs 3.7 months), and ORR (23\% vs $7 \%$ ) in favor of the combination compared with gemcitabine monotherapy [6].

So far, these chemotherapy regimens have not been directly compared, but indirect comparisons indicate greater efficacy (OS, ORR) of the FOLFIRINOX regimen with its greater toxicity. In addition, some patients eligible for treatment with the gemcitabine + nab-paclitaxel regimen are not eligible for FOLFIRINOX therapy. This group of patients is the subject of the Drug Program conducted in Poland since 2017.
Until recently, there was no clear evidence of the efficacy of II line chemotherapy. This changed the randomized phase III trial NAPOLI-1 published in 2018 [7]. Patients who progressed after treatment with gemcitabine-based regimens participated in the study. The efficacy of liposomal irinotecan in combination with 5-fluorouracil (5-FU) and leucovorin (LV) was verified compared to treatment with 5-FU/LV alone. The addition of liposomal irinotecan to 5-FU/LV extended the median OS (6.1 months vs 4.2 months), PFS (3.1 months vs 1.5 months), and ORR rates ( $17 \%$ vs $1 \%$ ) [7]. Guidelines of international scientific societies, including ESMO (European Society for Medical Oncology) recommendations, recognize liposomal irinotecan in combination with 5-FU/LV as a therapeutic option in the II line treatment for selected patients with metastatic pancreatic cancer [8].

In this paper, we present a case report of a 54-year-old patient with disseminated pancreatic cancer. From January 2018 to February 2020, he was treated with systemic chemotherapy with gemcitabine, nab-paclitaxel, 5-fluorouracil and nanoliposomal irinotecan.

\section{CASE REPORT}

In December 2017, a 54-year-old man with a tumor (largest transverse dimension $55 \mathrm{~mm}$ ) in the pancreatic head, without infiltration of the surrounding arterial or venous vessels, was referred to the Department of Oncological Surgery. The patient was qualified for an urgent resection. However, during the laparotomy, small nodular intraperitoneal dissemination was found. Resection of the primary lesion was abandoned, and biliointestinal and gastrointestinal anastomosis was performed on the Roux-en-Y loop. Histopathological examination confirmed the infiltration of adenocarcinoma of the pancreatic ductal adenocarcinoma in the peritoneal specimens taken. The patient was qualified for systemic treatment.

In January 2018, while qualifying for systemic treatment, the patient remained in a fairly good general condition - Karnofsky performance status (KPS) 70 , slight weight loss $(<10 \%$ in the last 3 months) and moderate pain persisted, requiring emergency use of non-opioid analgesics. The patient was treated chronically due to arterial hypertension. In addition, the patient had history of ischemic stroke 2 years ago (without neurological defects) and active smoking. The patient was finally qualified for the combination of gemcitabine with nab-paclitaxel.

After the first 2 months of therapy, imaging studies showed stabilization of the disease according to RECIST 1.1 (Response Eval- 
uation Criteria in Solid Tumors). Clinically, despite the necessity to reduce the dose of nab-paclitaxel due to the NCI-CTCAE grade II peripheral polyneuropathy (National Cancer Institute - Common Terminology for Adverse Events), improvement in general condition (KPS 90), stabilization of body weight and pain relief were observed. Pregabalin has been used to treat polyneuropathy. In addition, neutropenia and thrombocytopenia were observed during therapy, which required short-term treatment breaks and the use of growth factors. No grade IV toxicity according to NCI-CTCAE was noted. Low-molecular-weight heparins in prophylactic doses were used throughout the entire period of systemic therapy.

I line chemotherapy treatment was carried out until June 2019, when imaging studies revealed enlargement of peritoneal metastatic lesions meeting the RECIST 1.1 disease progression criteria. The patient remained in a good general condition, with no organ failure, and only with CTC grade I persistent polyneuropathy. No significant abnormalities were found in the blood count: neutrophils - $6.0 \mathrm{G} /$, lymphocytes - $1.62 \mathrm{G} / \mathrm{l}$, hemoglobin - $12.7 \mathrm{~g} / \mathrm{dl}$, platelets $-156 \mathrm{G} / \mathrm{l}$. The concentration of the CA 19-9 marker was $104 \mathrm{U} / \mathrm{ml}$. The patient was offered II line treatment according to the regimen of liposomal irinotecan + 5-FU/LV in standard doses. Dexamethasone, ondansetron and atropine were used as premedication. The therapy was started in July 2019. After the first 3 months, imaging studies continued to stabilize the disease according to the RECIST criteria, and a decrease in the CA 19-9 marker to $44 \mathrm{U} / \mathrm{ml}$ was also noted. The clinical tolerability of the treatment was good, with isolated episodes of diarrhea, neutropenia and fatigue not exceeding NCI-CTCAE grade II. Granulocyte colony stimulating factor (G-CSF) was used after each administration of chemotherapy for primary prophylaxis of febrile neutropenia. Not a single episode of febrile neutropenia has been reported.

In January 2020, after eight courses of chemotherapy, clinical signs of cancer progression were observed: ascites, weight loss, recurrence of pain, weakness. The imaging studies showed a progression of infiltrative changes within the peritoneum. Therefore, systemic treatment was discontinued and the patient was referred for symptomatic therapy. The patient died in April 2020, 28 months after starting systemic treatment.

\section{DISCUSSION}

About $40 \%$ of patients with disseminated pancreatic cancer who discontinue systemic therapy due to disease progression remain in good performance status [9]. From a clinical perspective, such patients would qualify for II line treatment, but until recently there was no clear evidence of the efficacy of II line chemotherapeutic agents. In patients treated in the I line with 5-FU-based regimens (including FOLFIRINOX), in clinical practice in the Il line, gemcitabine is most often administered as monotherapy, although there is no data from prospective, randomized clinical trials on the effectiveness of such treatment.

In patients who received gemcitabine-based regimens in I line therapy, 5-FU-based regimens are used in II line. Data on the combination of 5-FU and oxaliplatin are contradictory [10, 11]. The only prospective, randomized, phase III study unquestionably demonstrating the effectiveness of adding another chemotherapeutic agent to 5-FU/LV in the treatment of II line metastatic pancreatic cancer remains the NAPOLI-1 study [7].

It is worth noting that the patient described above achieved 18-month PFS with nab-paclitaxel plus gemcitabine and 7 months PFS with liposomal irinotecan in combination with 5-FU/LV. These results are better than the median PFS obtained in the MPACT and NAPOLI-1 studies, respectively. Also, the overall survival time, 28 months from the start of chemotherapy, is almost twice as long as the sum of the median OS in the registration studies mentioned.

In the post hoc analysis of the NAPOLI-1 study [12] conducted in 2019, factors of favorable prognosis were selected for the long-term survivors group, defined as patients who achieved at least one year of survival under II line treatment. In the NAPOLI-1 study, this was the group of $26 \%$ of patients in the nal-IRI + 5-FU/LV arm. Factors of a favorable prognosis include:

- age $\leq 65$ years

- Karnofsky perfomrance status $\geq 90$

- neutrophil to leukocyte ratio $(\mathrm{NLR}) \leq 5$

- no liver metastases

- CA 19-9 < 59 times upper limit of normal.

All these factors of a better prognosis were combined in this patient.

Treatment with nab-paclitaxel and gemcitabine is associated with a high risk of peripheral polyneuropathy, which develops at various levels in the majority of patients (over 50\%) [2]. In the presented case, the dose reduction of nab-paclitaxel and the use of pregabalin allowed for a permanent reduction of polyneuropathy symptoms to grade 1 according to NCI-CTCAE and the continuation of therapy until disease progression. A post hoc analysis of the MPACT study showed that the occurrence of polyneurop- 
athy was associated with longer survival [13]. It should therefore be emphasized that early diagnosis and appropriate interventions in the management of toxicity allow for the continuation of therapy and, consequently, prolongation of survival.

Due to the high clinical malignancy of pancreatic cancer, an extremely important element of the therapy is the time from the diagnosis of the disease to the initiation of appropriate treatment. In the case of the described patient, the time from laparotomy to the initiation of I line chemotherapy, as well as the time from the diagnosis of progression to the initiation of II line chemotherapy, did not exceed 4 weeks, which undoubtedly positively influenced the treatment results.

\section{CONCLUSION}

Treatment of disseminated pancreatic cancer with the use of two lines of systemic chemotherapy in selected groups of patients allows for long-term clinical response to treatment.

\title{
References
}

1. Siegel RL, Miller KD, Jemal A. Cancer statistics, 2016. CA: Cancer J Clin. 2016; 66: 7-30

2. Oettle H, Corrie P, Reni M et al. Pancreatic cancer. Nab-Paklitaksel. Börm Bruckmeier Verlag 2015: 6.

3. SEER. Cancer Stat Facts: Pancreatic Cancer. http://seer.cancer.gov/statfacts/html/pancreas.html (access: 1.02.2021).

4. Burris HA 3rd, Moore MJ, Andersen J et al. Improvements in survival and clinical benefit with gemcitabine as first-line therapy for patients with advanced pancreas cancer: a randomized trial. J Clin Oncol. 1997; 15(6): 2403-13.

5. Conroy T, Desseigne F, Ychou M et al. FOLFIRINOX versus gemcitabine for metastatic pancreatic cancer. N Engl J Med. 2011; 364(19): 1817-25.

6. Von Hoff DD, Ervin T, Arena FP et al. Increased survival in pancreatic cancer with nab-paclitaxel plus gemcitabine. N Engl J Med. 2013; 369(18): 1691$-703$.

7. Wang-Gillam A, Li CP, Bodoky G et al. Nanoliposomal irinotecan with fluorouracil and folinic acid in metastatic pancreatic cancer after previous gemcitabine-based therapy (NAPOLI-1): a global, randomised, open-label, phase 3 trial. Lancet. 2016; 387(10018): 545-57.

8. Ducreux M, Cuhna AS, Caramell C et al. ESMO Guidelines Committee: Cancer of the pancreas: ESMO Clinical Practice Guidelines for diagnosis, treatment and follow-up. Ann Oncol. 2015; 26(suppl 5): v56-v68.

9. Hegewisch-Becker S, Aldaoud A, WolfT et al. International Journal of Cancer. July 2018 Results from the prospective German TPK clinical cohort study: Treatment algorithms and survival of 1,174 patients with locally advanced, inoperable, or metastatic pancreatic ductal adenocarcinoma. Int J Cancer. 2019; 144(5): 981-90.

10. Oettle H, Riess J, Stieler JM et al. Second-line oxaliplatin, folinic acid, and fluorouracil versus folinic acid and fluorouracil alone for gemcitabine-refractory pancreatic cancer: outcomes from the CONKO-003 trial. J Clin Oncol. 2014; 32: 2423-9.

11. Gill S, Ko Y-j, Cripps C et al. PANCREOX: a randomized phase III study of 5 fluorouracil/leucovorin with or without oxaliplatin for second-line advanced pancreatic cancer in patients who have received gemcitabine-based chemotherapy. J Clin Oncol. 2016; 4(32): 3914-20.

12. Wang-Gillam A, Hubner RA, Siveke JT et al. NAPOLI-1 phase 3 study of liposomal irinotecan in metastatic pancreatic cancer: Final overall survival analysis and characteristics of long-term survivors. Eur J Cancer. 2019; 108: 78-87.

13. Goldstein D, Von Hoff DD, Moore M et al. Development of peripheral neuropathy and its association with survival during treatment with nab-paclitaxel plus gemcitabine for patients with metastatic adenocarcinoma of the pancreas: A subset analysis from a randomised phase III trial (MPACT). Eur J Cancer. 2016; 52: 85-91.

\author{
Authors' contributions: \\ All authors contributed equally to the article. \\ Conflict of interests: \\ Authors declare no conflict of interest. \\ Financial support: \\ None. \\ Ethics: \\ The authors had full access to the data and take full responsibility for its integrity. \\ All authors have read and agreed with the content of the manuscript as written. \\ The paper complies with the Helsinki Declaration, EU Directives and harmonized requirements for biomedical journals.
}

